\title{
Adaptive Modulation and Coding with Cooperative Transmission in MIMO fading Channels
}

\author{
Yuling Zhang ${ }^{1, \text { a }}$, Qiuming $\mathrm{Ma}^{2, \mathrm{~b}}$ \\ ${ }^{1}$ School of Information and Electrical Engineering, Ludong University, Yantai, Shandong, 264025, \\ P. R. China \\ ${ }^{2}$ School of Information and Electrical Engineering, Ludong University, Yantai, Shandong, 264025, \\ P. R. China \\ aemail: zhang-yuling@hotmail.com, bemail: mqml@sina.com
}

Keywords: Cooperative transmission, adaptive modulation and coding (AMC), multiple-input multiple-output (MIMO) channels.

\begin{abstract}
In this paper, we propose a cooperative transmission framework based on adaptive modulation and coding (AMC) system in multiple-input multiple-output (MIMO) fading channels. We consider the scenario that consists of one source node, one relay node and one destination node, they all equipped with multiple antennas and employed the adaptive modulation and coding schemes. Through computer simulation, we got the effective capacity of the adaptive cooperative transmission system according to different QoS requirement.
\end{abstract}

\section{Introduction}

The increasing development of wireless applications, especially real-time media traffic with stringent QoS constraints requires a high efficient utilization of the scarce radio resources. Many techniques are proposed to improve the throughput of time-varying fading channels while maintaining a satisfied QoS, such as link adaptation based on adaptive modulation and coding (AMC) and automatic repeat request (ARQ) [1][2][3], multiple-input and multiple-output systems and cooperative transmission through node cooperation.

There are many literature about the adaptive cooperative transmission, Jalil Seifali investigates the effective capacity for multi-rate relay channels with delay constraint exploiting adaptive cooperative diversity [4], and authors in [5] design a decision-making algorithm on cooperative transmission by using a partially observable Markov decision process framework. Shan Chu exploits the use of cooperative relay transmission in a MIMO-based ad hoc network to cope with harsh channel condition [6]. In this paper, we propose a framework of adaptive cooperative transmission based on adaptive modulation and Low-density parity-check (LDPC) codes in multi-antenna systems.

The rest of this paper is organized as follows. In Section II, we present the system model and illustrate the principle of the cooperation transmission. How to calculate the effective capacity are explained in Section III. In Section IV, we get numerical results through simulations. Finally, some conclusions are drawn.

\section{System Model}

The system model of cooperative transmission based on LDPC codes in MIMO channels is shown in Fig.1. The system consists of source node $\mathrm{S}$, destination node $\mathrm{D}$ and relay node $\mathrm{R}$. Assuming that there are $N_{T}$ transmit antennas, $N_{R}$ relay antennas and $N_{\mathrm{D}}$ receive antennas.

The adaptive transmission is achieved by two means, at the physical layer, there are multiple modulation and coding schemes (MCSs) available, not only in source node but also in relay node. Coded symbols are transmitted to the relay node and the destination node simultaneously on a frame-by-frame basis through MIMO fading channels after space-time block coding. The CSI is estimated at the receiver and then sent back through a feedback channel to the AMC controller for both the source node and delay node, which chooses the appropriate MCS in the next transmission 
accordingly.

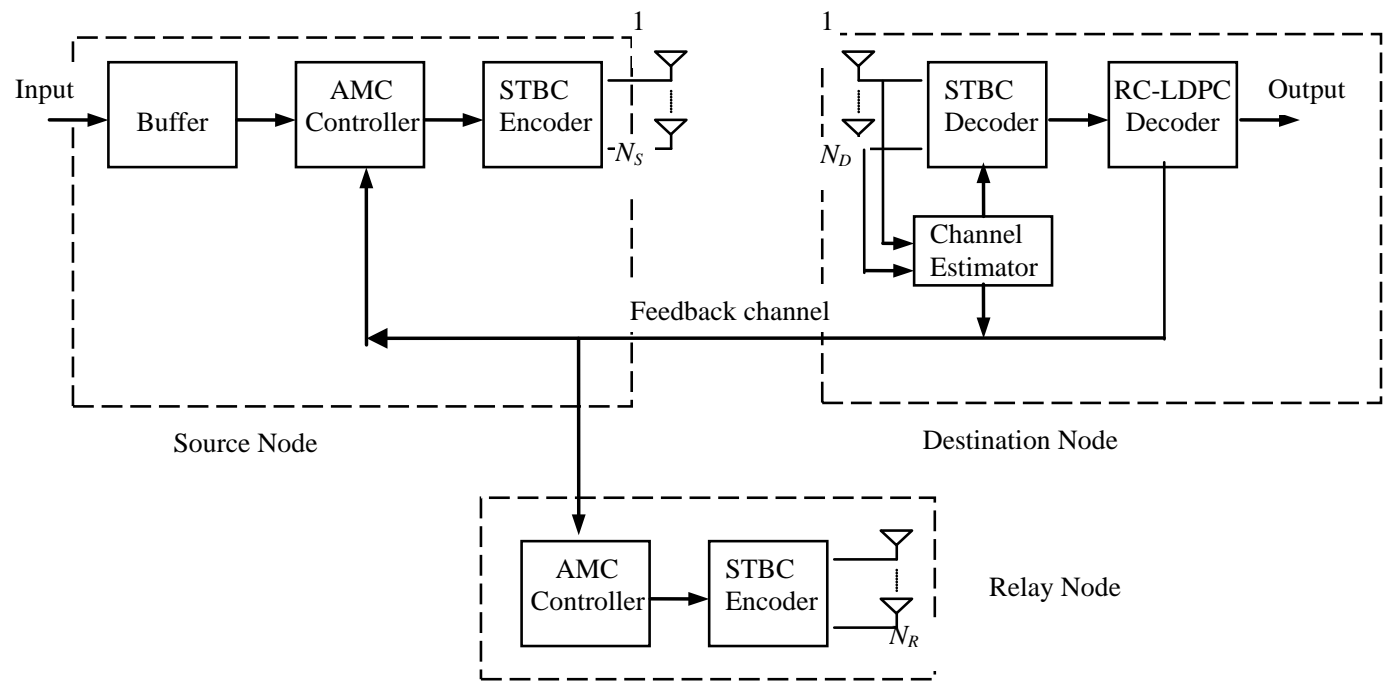

Fig. 1. System model

At the data link layer, adaptive cooperative retransmission is employed to improve the throughput performance, especially for the communication of delay-sensitive packet traffic. The packet and frame structures used in this paper are similar to those as illustrated in [1]. Source information are transmitted frame by frame, each frame is divided into two time-slots, during the first time-slot, node S selects an AMC mode based on the S-D channel condition, meanwhile node $\mathrm{R}$ and node $\mathrm{D}$ listen. When an error is detected in a packet at node $\mathrm{D}$, a retransmission request is generated and sent back to node $\mathrm{S}$ and node $\mathrm{R}$ via a feed back channel. Then at the second time-slot, node $\mathrm{R}$ forwards the packet received from node $\mathrm{S}$ to node $\mathrm{D}$ when it is able to decode the source packet correctly, otherwise, node S will deliver the packet again. For simplicity, we assume that the feed back channel is error free and has zero delay.

A block fading channel model is adopted for all S-D, S-R,and S-D links. Here we take the S-D link for an example, S-R and S-D links can be analyzed in a similar way. The MIMO fading channel between $\mathrm{S}$ and R can be expressed as a matrix $\mathbf{H}_{S R}=\left[h_{i j}\right]_{i, j=1}^{N_{S}, N_{R}}$, where $h_{i j}$ is the channel coefficient between the $j$ th transmit antenna and the ith relay antenna. Under the assumption of independent Rayleigh fading, the channel coefficients $h_{i j}$ are modeled as independent and identically distributed (i.i.d.) complex circular Gaussian random variables with zero mean and unit variance. According to the effective SISO channel model for STBCs described in [7],the SNR at the receiver is given by

$$
\gamma_{1}=\frac{P_{s} d_{1}^{-\alpha}}{\sigma^{2}}\left\|\mathbf{H}_{S R}\right\|_{\mathrm{F}}^{2}=\frac{P_{T} d_{1}^{-\alpha}}{\sigma^{2} N_{T} R_{c}}\left\|\mathbf{H}_{S R}\right\|_{\mathrm{F}}^{2}=\frac{\overline{\gamma_{1}}}{N_{T} R_{c}}\left\|\mathbf{H}_{S R}\right\|_{\mathrm{F}}^{2}
$$

where the coding rate of STBC is $R_{c}=R / T, P_{s}$ is the average transmit power per stream/antenna, $P_{T}$ is the total transmit power transmitted on $N_{T}$ antennas per symbol duration and $\bar{\gamma}_{1}=P_{T} d_{1}^{-\alpha} / \sigma^{2}$ is defined to be the average pseudo SNR, in which $d_{1}$ is the Euclidean distances between noses $\mathrm{S}$ and D, $\alpha$ is the path loss exponent. Since $\left\|\mathbf{H}_{S R}\right\|_{\mathrm{F}}^{2}$ is the sum of $2 K$ i.i.d. $\chi^{2}$ random variables, we can get the probability density function (PDF) of $\gamma_{1}$ as follows [8]

$$
p_{\gamma_{1}}\left(\gamma_{1}\right)=\frac{\gamma_{1}^{K-1}}{\Gamma(K)}\left(\frac{N_{T} R_{c}}{\gamma_{1}}\right)^{K} \exp \left(-\frac{N_{T} R_{c}}{\gamma_{1}} \gamma_{1}\right), \quad \gamma_{1} \geq 0
$$

where $\Gamma(\cdot)$ is the Gamma function.

In the same way, we let $\gamma_{2}$ and $\gamma_{3}$ denote the received SNR on the R-D, and S-R channels, the Euclidean distances between noses $\mathrm{R}$ and $\mathrm{D}$, and noses $\mathrm{S}$ and $\mathrm{R}$ are $d_{2}$ and $d_{3}$ respectively, the average SNRs are given by $\overline{\gamma_{2}}=P_{r} d_{2}^{-\alpha} / \sigma^{2}$, and $\overline{\gamma_{3}}=P_{T} d_{3}^{-\alpha} / \sigma^{2}$.

The AMC schemes adopted at node $\mathrm{S}$ and node $\mathrm{R}$ can be illustrated as follows: Packets received 
incorrectly after $N_{r}$ retransmissions will be dropped. In order to meet the system delay constraint, for a given packet loss probability $P E R_{\text {link }}$ at the data link layer, the packet error rate (PER) $P_{\text {target }}$ at the physical layer should be [1]

$$
P_{\text {target }}=P E R_{\text {link }}^{1 /\left(N_{r}+1\right)}
$$

The AMC is implemented at the physical layer according to the target PER. Suppose that there are $N$ MCSs at the physical layer with increasing rates $R_{n}(n=1,2, \ldots, N)$ in terms of information bits per symbol. We will consider the modulation method with the MQAM signal constellation, where $M$ denotes the number of points in each signal constellation. If the coding rate of a MCS is $R_{L}$, we have $R_{n}=R_{L} \cdot\left(\log _{2} M\right)$. As in [1], the whole range of the SNRs $\gamma_{1}, \gamma_{2}$ are divided into $N+1$ and $M+1$ non-overlapping consecutive intervals, denoted by $\left[\gamma_{n, 1}, \gamma_{n+1,1}\right), n=0,1, \ldots N$, and $\left[\gamma_{m, 2}, \gamma_{m+1,2}\right), m=0,1, \ldots M$. When $\gamma_{1}$ falls into the interval $\left[\gamma_{n, 1}, \gamma_{n+1,1}\right), n \geq 1$, node $\mathrm{S}$ selects the AMC mode $\mathrm{n}$ and sends data with transmission rate $R_{n, 1}$ (bits/symbol). In a similar way, when $\gamma_{2} \in\left[\gamma_{m, 2}, \gamma_{m+1,2}\right), m \geq 1$, node $\mathrm{R}$ selects mode $\mathrm{m}$ and sends data to node $\mathrm{D}$ with rate $R_{m, 2}$ (bits/symbol). When $\gamma_{2} \in\left[\gamma_{0,2}, \gamma_{1,2}\right)$, no data is sent by node R. But when $\gamma_{1} \in\left[\gamma_{0,1}, \gamma_{1,1}\right)$, nodes $\mathrm{S}$ still transmits packets from its buffer with the first AMC mode $(\mathrm{n}=1)$.

The thresholds $\gamma_{n, 1}$ and $\gamma_{m, 2}$ are determined in the same way as in [2], according to the AMC rule, each MCS $n$ will be chosen with the following probability [1]

$$
p_{n, 1}=\int_{\gamma_{n, 1}}^{\gamma_{n+1,1}} p_{\gamma_{1}}\left(\gamma_{1}\right) d \gamma=\frac{\Gamma\left(K, \lambda \gamma_{n, 1}\right)-\Gamma\left(K, \lambda \gamma_{n+1,1}\right)}{\Gamma(K)} .
$$

It can be shown that the average PER for MCS $n$ is given by

$$
\overline{\operatorname{PER}}_{n, 1}=\int_{\gamma_{n, 1}}^{\gamma_{n+1,1}} \operatorname{PER} R_{n}\left(\gamma_{1}\right) p_{\gamma_{1}}\left(\gamma_{1}\right) d \gamma_{1} \text {. }
$$

Then, the total average PER can be written as follows

$$
\bar{P}_{n, 1}=\frac{\sum_{n=1}^{N} R_{n, 1} \overline{\mathrm{PER}}_{n, 1}}{\sum_{n=1}^{N} R_{n, 1} p_{n, 1}} .
$$

In real time multimedia services such as video transmission, time delay is an important QoS parameter. Effective capacity (EC) is proposed to describe the maximum throughput of the system under delay constraint, and widely used to analyze the QoS performance of wireless multimedia networks.

In Rayleigh fading channel, let the binary random variable $X_{\mathrm{i}}$ indicate the number of packets serviced by the queue service process at node $S$ in frame i. If the packet in the ith frame is decoded correctly by node $\mathrm{D}$ then $X_{i}=1$, otherwise $X_{i}=0$ (packet error). The EC function can be upper-bounded as follows

$$
E C(\theta) \leq \frac{-1}{\theta \bar{T}_{f}} \log \left[E_{X_{i}}\left\{\exp \left(-\theta N_{b} X_{i}\right)\right\}\right]=\frac{-1}{\theta \bar{T}_{f}} \log \left[\exp \left(-\theta N_{b}\right)+\left(1-\exp \left(-\theta N_{b}\right)\right) \operatorname{Pr}\left(X_{i}=0\right)\right]
$$

Where $\theta \geq 0$ reflects the quality requirement of the transmission [9]. A smaller $\theta$ represents a looser QoS constraint, when $\theta$ tends to 0 , an arbitrarily long delay can be tolerated, the EC converges to the maximum throughput (ergodic capacity) of the system.

In an AMC-based transmission system with a fixed symbol rate, the duration of each time slot in a frame depends on the employed AMC mode. If in the first time-slot, node S transmits with mode $\mathrm{n}$, the duration of this time-slot is denoted by

$$
T_{s, 1}^{(n)}=\frac{N_{b}}{R_{n, 1} R_{s}}, \quad \forall n \geq 1
$$

Where $N_{b}$ is the packet length in bits, and $R_{s}$ is the channel symbol rate per second. Similarly, the retransmission time duration in the second time-slot for node $\mathrm{R}$ can be expressed as 


$$
\begin{aligned}
& T_{s, 2}^{(m)}=\frac{N_{b}}{R_{m, 2} R_{s}}, \quad \forall m \geq 1 \\
& \operatorname{Pr}\left(X_{i}=0\right)= \\
& p_{0,1} I_{1} \overline{P E R}_{0,1}+\sum_{n=1}^{N} p_{n, 1} I_{n} \overline{P E R}_{n, 1}+\left(p_{0,1}\left(1-I_{1}\right) \overline{P E R}_{0,1}+\sum_{n=1}^{N} p_{n, 1}\left(1-I_{n}\right) \overline{P E R}_{n, 1}\right)\left(p_{0,2}+\sum_{m=1}^{M} p_{m, 2} \overline{P E R}_{m, 2}\right) \\
& p_{m, 2}=\int_{\gamma_{m, 2}}^{\gamma_{m+1,2}} p_{\gamma_{2}}\left(\gamma_{2}\right) d \gamma=\frac{\Gamma\left(K, \lambda \gamma_{m, 2}\right)-\Gamma\left(K, \lambda \gamma_{m+1,2}\right)}{\Gamma(K)} \\
& I_{n}=\int_{0}^{\infty} P E R_{n}\left(\gamma_{3}\right) p_{\gamma_{3}}\left(\gamma_{3}\right) d \gamma_{3}
\end{aligned}
$$

for the relay-assisted transmission system, the expected value of the frame length $\bar{T}_{f}$ is given by

$$
\bar{T}_{f}=E\left(T_{f}^{(i)}\right)=\sum_{n=0}^{N} T_{s, 1}^{(n)} \bar{P}_{n, 1}+\left(\sum_{m=1}^{N} T_{s, 2}^{(m)} p_{m, 2}\right) \times\left(p_{0,1}\left(1-I_{1}\right) \overline{P E R}_{0,1}+\sum_{n=1}^{N} p_{n, 1}\left(1-I_{n}\right) \overline{P E R}_{n, 1}\right)
$$

\section{Simulation Results}

In this section, numerical results showing the effects of different parameters on the EC of our cooperative transmission framework are provided. Firstly, the system parameters are set as follows: The S-D distance $d_{1}$ is normalized to one, the relay position is controlled by changing the R-D distance $d_{2}$, path loss exponent $\alpha=4$, the packet length is $N_{b}=1008$ bits. The frame time duration in the AMC mode $1, T_{s, 1}^{(1)}=2 m s$, relay position $d_{2}=d_{3}=d_{1} / 2$, equal transmit powers for nodes $\mathrm{S}$ and R. Nodes S and R use the same AMC mode set, adopting from the IEEE 802.11a standard [10], we use RC-LDPC codes instead of convolutional codes. Assuming that the performance constraint at the data link layer is $P E R_{\text {link }}=0.01$. The variable node degree distribution of irregular LDPC codes is as follows

$$
\lambda(x)=\sum_{i} \lambda_{i} x^{i}=0.47532 x^{2}+0.27953 x^{3}+0.03486 x^{4}+0.10889 x^{5}+0.10138 x^{15}
$$

The EC for both cooperative and direct transmission systems under several channel scenarios is given in Fig.2. In noncooperative transmission scheme, node $S$ directly transmit its packets to node $\mathrm{D}$ without the assistance of the relay node. We can see in fig.2, compared with the noncooperative transmission, the cooperative protocol dramatically improves the EC.

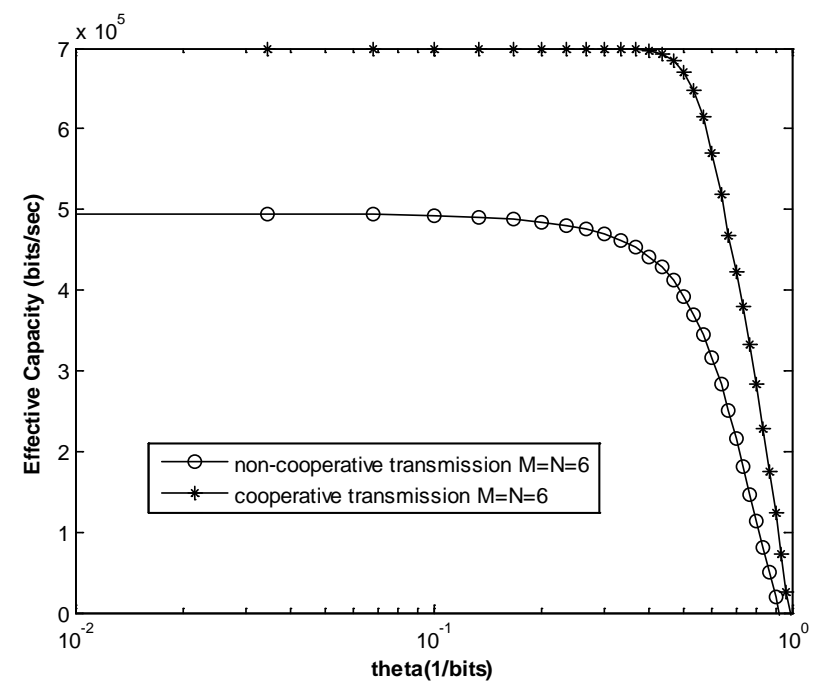

Fig.2 EC for different $\theta$

\section{Conclusion}

In this paper, we proposed an adaptive transmission frame in cooperative communication systems under 
MIMO channel. The AMC at the physical layer and the adaptive cooperative transmission at the data link layer are combined to achieve a better EC. At the source node and the relay node, relevant MCS is chosen based on the SNR thresholds calculated according to the LDPC PER-SNR relationship. Numerical results show that the cooperative system can provide better EC than the non-cooperative system.

\section{Acknowledgment}

This work is supported by National Scientific Foundation of China (No. 61273152, No.61471185) and Scientific Foundation of Shandong Province (No. ZR2013FL007).

\section{References}

[1] Q. Liu, S. Zhou, and G. B. Giannakis. Cross-layer combining of adaptive modulation and coding with truncated ARQ over wireless links [J]. IEEE Transactions on Wireless Communications, 2004,3 (5): 1746-1755.

[2] Yuling Zhang, Dongfegn Yuan, Chengxiang Wang, Cross-Layer Design Based on RC-LDPC Codes in MIMO Channels with Estimation Errors [J]. AEU - International Journal of Electronics and Communications, 2008, 62(9): 659-665.

[3] D. Wu and R. Negi, Effective Capacity: a wireless link model for support of quality of services. IEEE transactions on Wireless Communications [J]. July 2003, 2(4):630-643.

[4] Jalil Seifali Harsini and Michele Zorzi. Effective Capacity for Multi-Rate Relay Channels with Delay Constraint Exploiting Adaptive Cooperative Diversity [J]. IEEE Transactions on Wireless Communication, 2012, 11(9): 3136-3147.

[5] Dong Heon Lee, Wha Sook Jeon, Kae Won Choi etc. Adaptive transmission policy over Rayleigh fading channels for cooperative networks with limited feedback [J]. IET Communications, 2013, 7(17): 1907-1914.

[6] Shan Chu ; Xin Wang ; Yuanyuan Yang. Exploiting Cooperative Relay for High Performance Communications in MIMO Ad Hoc Networks [J]. IEEE Transactions on Computers, 2013, 62(4): 716-729.

[7] V. Tarokh, H. Jafarkhani, and A. R. Calderbank. Space-time block codes from orthogonal designs [J]. IEEE Trans. Inform. Theory, 1999, 45 (5): 1456-1467.

[8] D. Lu. Stochastic Process and Its Application. in Chinese, Tsinghua University Press, 1986.

[9] L.Liu, P.Parag, and J.-F Chamberland. Quality of service analysis for wireless user-cooperation networks. IEEE Trans. Inform. Theory, October 2007, 53(10):3833-3842.

[10] A. Doufexi, S. Armour, M. Butler, A. Nix, D. Bull, J. McGeehan, and P. Karlsson. A comparison of the HIPERLAN/2 and IEEE 802.11a wireless LAN standards [J]. IEEE Communications Magazine, 2002, 40 (5): 172-180. 